\title{
An Analysis of the Benfit on Green Risk in Construction Projects
}

\author{
Xianfeng Zhang, Chuanmin Shuai \\ School of E\&M, China University of Geosciences, Wuhan, China. \\ Email: tobyzhang@163.com
}

Received May $18^{\text {th }}, 2010$; revised June $9^{\text {th }}, 2010$; accepted June $18^{\text {th }}, 2010$.

\begin{abstract}
The Construction project green risks (CPGRs) refer to those threats to environment, energy sources and material resources during the entire life-cycle of a construction project. The emergent green risks in exploiting these resources are of varying concern to all. In this paper, evolutionary game is introduced to make about impacts of strategy choices from interactions among the choices developers, and between the choices developers and EPDs on project green risk. The results show that CPGRs will occur if either developers find that not managing CPGRs has a better payoff than opting for CPGR management, or if monitors impose only mild fines even when they find CPGRs within construction projects of developer's firms. The study also shows that there is a prohibitively expensive cost incurred by EPDs in monitoring CPGRs. Finally, some strategies are given for EPDs to help them make policies to regulate the strategies of developers.
\end{abstract}

Keywords: Construction Project Management, Green Risk, Evolutionaryary Game, Strategy Developer

\section{Introduction}

\subsection{Construction Project Green Risk}

Construction is a process of building in which materials are transformed into products, e.g. buildings, airports and highways, which inevitably leads to some form of environmental pollution, energy consumption and resource depletion. Up to date Chinese government has called for "energy-saving and pollution-reducing" initiatives. Such a policy is helpful to prod construction project green risk developer (CPGR-developers) to have concern for the environment and to manage green risks. However, the validity of such policies has yet to not be seen.

Green risks (GRs) are those threats to human beings and/or to what they value, from hazards, either natural or human-driven, associated with global change [1]. CPGRs refer to those threats to environment, energy and resources which occur during the entire life cycle of construction project, and the process is depicted as Figure 1.

\subsection{Brief View of CPGRs Researches}

A necessary question to pose is how to raise awareness toward CPGRs and with collaboration of the construction sector to promote solutions that better conservation policies. There two viewpoints about CPGRs, and some researchers hold views that lowering CPGRs can resort to

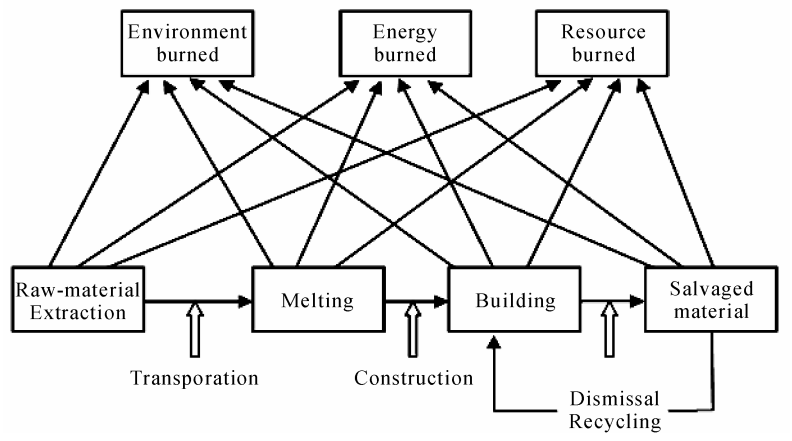

Figure 1. The impacts on environment, resource and energy.

reducing building materials consumption [3-11]; but others thought although using less material can avert CPGRs yet this response could sacrifice some building functions, and so they suggested an alternative approach based on boosting the cost of introducing CPGRs, which advocating government or EPDs taxing or penalising much more than previous if an enterprise creates or elevates CPGRs, such as raising the resource production price, reducing the overall energy amount to be used by an enterprise, penalising pollution violators, to block the projects from proceeding. Further they have analysed CPGRs based on game theory [12-14], and believed that heavy fines will encourage construction managers to deal with green risk, 
compel them to make a decision either to comply with environmental policy decreed by government to better management of CPGRs, or to face penalties from EPDs and incur financial cost or some other such punishment.

Unfortunately traditional game theory is usually limited to exploring rational agents' interactions. However, the rational assumption is not valid for CPGR-developers in that these developers will find it difficult to know competitors' strategies and hence their own decisions will not be treated rationally due to incomplete knowledge. Thus bounded rationality, as it is called, is the norm, and over time CPGR-developers will learn and develop their own strategies, or imitate other players' strategies. Indeed, they may change them constantly and hence develop an evolutionary stable strategy (ESS). In this article, evolutionary game is introduced to analyse the choice behaviours, by considering decision-makings of CPGR developers and their interactions among each other and with EPDs, in order to find motives and reasons to behaviours towards CPGRs.

\section{The Evolutionary Game Based Model of CPGR Developers}

Confronted with CPGRs, developers (those firms who by their activity in the construction area produce the CPGRs) have two choices. One choice, which here is called manag, is using new technology to reduce green risk. The technology may take the form of utilizing low-energy consumption machines, providing low-energy steel, or increasing the use of recycled metals. The other choice is non-manag, i.e., in plain speak, shirking responsibility for green risk and simply doing nothing. Now we assume that there exists a construction project for which there are two vying construction firms that have presented project tenders. Within the game theory context, we introduce outcomes determined by the attitude of the developers to CPGRs. If a developer elects to deal with them the gain is $A$; if the other does nothing, the developer gets $B$, otherwise $C$. Of course, the two developers will both get $D$ if they show no concern toward CPGRs. The payoff matrix that game theory ascribes to the two players of this game is as follows Table $\mathbf{1 .}$

For the two players, the problem is which strategy should be employed; the decision depends on the action

Table 1. Payoff matrix of CPGR-developers.

\begin{tabular}{cccc}
\hline & & \multicolumn{2}{c}{ CPGR-Developer II } \\
\hline & & Manag. & Non-manag. \\
CPGR-Developer I & Manag. & $\boldsymbol{A}, \boldsymbol{A}$ & $\boldsymbol{B}, \boldsymbol{C}$ \\
& Non-manag. & $\boldsymbol{C}, \boldsymbol{B}$ & $\boldsymbol{D}, \boldsymbol{D}$ \\
\hline
\end{tabular}

of the other; such a situation illustrates a game of imperfect information. As a consequence, we rank the pay-offs as $A \neq B \neq C \neq D$. If two players are rational economic agents, the result of the game depends on the relative values assigned to $A, B, C$ and $D$. However, the developers are in fact bounded rational agents, so after playing the game, the values $A, B, C$ and $D$ must have some relevance to reality, so that evolutionary game theory is applicable.

In this game, the two developers choose between two strategies with a certain payoff. However, how will a population of developers that repeatedly play this game evolve? In what follows, it will be assumed that the payoffs will be the same for every developer. We cannot answer the above question without introducing some assumptions concerning the nature of the population. Let us being by assuming the number of developers is large, so that we can represent the state of the population by keeping tract of what proportion of developers follow the strategy management and those following non-management. We further assume that the proportions following a particular strategy at the next generation of play is proportional to that of the current generation. Thus the strategies themselves are now playing each other. This then provides us with differential equations and hence continuous dynamics known as replicator dynamics for an evolutionary gaming theory. Finally we assume that strategies are uncorrelated, i.e. that the probability with which every strategy meets every other strategy depends only on the relative frequencies within the population. Thus the games between developers are randomly played.

We denote the frequency of population in management strategy in the CPGR decision game as $x$, which will vary with time $t$, and consequently the population ratio of non-management strategies with $1-x$, and then We have:

$$
\left\{\begin{array}{l}
u_{t}=x A+(1-x) B \\
u_{f}=x C+(1-x) D \\
\bar{u}=x u_{t}+(1-x) u_{f}
\end{array}\right.
$$

where $u_{t}$ is the expected gain for the management strategy, $u_{f}$ is the expected gain for the non-management strategy, $\bar{u}$ is the average gain for all construction firms for the project at that generation.

Based on $2 \times 2$ symmetric game model, we can get dynamic Equation (2), then obtain Equation (3) if substituting (1) into, and further induce Equation (4) and three possible stationary states are eventually obtained if setting $F(x)=d x / d t=0$. According to evolutionaryary stable strategy (ESS) of the differential equation, we obtain the optimal solution $x_{i}{ }^{*}$ is ESS, if $F^{\prime}\left(x_{i}^{*}\right)=0$. The equations listed as follows: 


$$
\begin{gathered}
d x / d t=x\left(u_{t}-\bar{u}\right)=x(1-x)\left(u_{t}-u_{f}\right) \\
F(x)=d x / d t=x(1-x)[x(A-C)+(1-x)(B-D)] \\
\left\{\begin{array}{l}
x_{1}^{*}=0, x_{2}{ }^{*}=1, \\
x_{3}{ }^{*}=\frac{D-B}{A-B-C+D} \text { if } 0 \leq \frac{D-B}{A-B-C+D} \leq 1
\end{array}\right.
\end{gathered}
$$

In the model there are four aspects needed to be focused as follows:

The circumstance $A=C$ and $B=D$ implies one player, who is playing management strategy, will receive more gain, irrespective of whether the other players are executing management strategy or not. This situation will occur if government can strictly enforce laws concerning CPGR-developers. If this is the case, the risks can be determined and developers will be punished. The loss resulting from punishment is higher than the cost of managing the risks. Thus, $F^{\prime}\left(x_{1}^{*}\right)=0, F^{\prime}\left(x_{2}^{*}\right)<0, x_{3}^{*}$ is not ESS, $x_{2}^{*}=1$ is the only ESS. Therefore, we can describe the game result as one in which a bounded rational developer, after playing repeatedly, will change to management strategy. This selection will encourage management strategy as the cleverer scheme for developers.

It is possible that one player who opts for non-management strategy will gain more whether or not it is played by the other player. This situation requires $A<C$ and $B$ $<D$. Normally, this state arises when the EPDs are completely in breach of their duty and hence the ignored CPGR-developers will find that there would be more gain if they played non-management strategy rather than management strategy. In this case $x^{*}{ }_{1}=0$ is the only ESS because $F^{\prime}\left(x_{1}^{*}\right)<0$ and $F^{\prime}\left(x_{2}^{*}\right)=0$. Playing the game repeatedly, bounded rational developers will think it is foolish to elect management strategy.

If one player chooses management strategy in this CPGR decision game, the other player then finds management strategy is a smart choice for himself, as in this situation if $A=C$ and $B<D$ is satisfied then one player receives less gain from management strategy than from the non-management strategy when the other player does nothing about CPGRs. These cases will occur repeatedly if the non-management action earns more benefit than the loss incurred in the punishment from EPDs. Of course, $F^{\prime}\left(x_{1}{ }_{1}\right)<0, F^{\prime}\left(x_{2}{ }_{2}\right)<0, F^{\prime}\left(x_{3}{ }_{3}\right)=0, x^{*}{ }_{1}=0$ and $x_{2}^{*}=1$ are ESSes. Thus the game result will decided on the original population level of $x$. When $x \in\left(0, x_{3}^{*}\right)$, after repeated play, the developer will give up management strategy. However, when $x \in\left(x_{3}^{*}, 1\right)$, the contrary situation will be taken up. Especially, if $D=B, A \neq C$, thus $x_{3}^{*}=0$, all the developers will chose non-management plan and the management strategy is used when $D \neq B$ and $A=C$ because of $x_{3}^{*}=1$.

Of course, if $A<C$ and $B=D$ then this situation implies that management strategy has less gain than nonmanagement when the other developer executes management strategy, or means the contrary strategy when the others abandon management strategy while the player finds that he will get severely punished due to increasing CPGRs. This means the player's strategy of choice depends on the other players' actions according to the seriousness of CPGRs. For example, after using non-management strategy, developers find themselves receiving less fines and the others playing management to the risk resource at the same time. Which choices will they make? Clearly, non-management strategy! In contrast, when they find they are receiving higher fines, the only right choice is to take up management strategy because if they give up management the nasty risk resources strategy within the others doing the same things. Thus $F^{\prime}\left(x^{*}{ }_{1}\right)>0, F^{\prime}\left(x_{2}^{*}\right)>0, F^{\prime}\left(x^{*}{ }_{3}\right)<0, x^{*}{ }_{3}$ is the only ESS. The result tells us the frequency $x_{3}{ }_{3}$ of developers out of all developers will management the CPGRs after repeatedly playing the game, and the frequency playing non-management strategy will be $\left(1-x_{3}^{*}\right)$. Obviously, the frequency of management strategy players is rising with " $B-D$ " increasing, but increasing " $C-A$ " attracts some bad implications.

From the above analysis, we know that projects receiving little CPGR is possible if we reduce the expected benefit of non-management strategy over management strategy. Therefore, serious policy is necessary from government. In the next section, we discuss game strategies between monitors from environment protection departments (EPD) and CPGR-developers.

\section{The Evolutionary Game Based Model of CPGR-Developers and EPDs}

The developers have two choices: implementing either a management or non-management strategy in confronting CPGRs; meanwhile the EPDs can also chose between a monitoring or non-monitoring attitude. Through environmental monitoring, EPDs will provide judgment on whether CPGRs are acceptable, in which case no action is taken, or unacceptable, in which case a fine will be adjudicated. However monitoring needs funding and may even cost more than is bargained, thus creating in itself a problem for EPDs. In contrasting the comparative gain between various strategies, we assume the benefit to two players is 0 (in reality, the benefit is not 0 ) under the criterion that developers will execute a management strategy toward CPGRs and the EPD carries out non-monitor strategy. We then let $C_{1}>0$ refer to the EPD's monitoring costs of CPGRs, $C_{2}>0$ the punishment to EPDs for breach of duty from government, $S>0$ the fine to be imposed by EPDs on non-management behaviour of 
firms, and $R \geq 0$ the incentives or bonuses to be awarded from government to EPDs for good monitoring behaviours.

The payoff matrix for EPDs and CPGR-developers is shown in Table 2. EPDs and CPGR-developer are assumed to be bounded rational agents. We define $x$ as the frequency of management strategy in the developer population and define $y$ as the frequency of monitoring strategy in the EPD population. We also define $u_{1 t}$ as the expected gain for a management strategy, $u_{1 f}$ as the expected gain for non-management strategy, $u_{1}$ as the average gain for all construction firms at a given generation, $u_{2 t}$ is the expected gain for monitoring strategy, $u_{2 f}$ is the expected gain for non-monitoring strategy, $u_{2}$ is the average gain for all EPDs at a given generation.

The replicator dynamic equation for developers and EPDs are respectively expressed as Equations (5) and (6) as following:

$$
\begin{gathered}
F(x)=d x / d t=x(1-x)(S-A-S y) \\
\left.G(y)=d y / d t=y(1-y)\left[C_{1}-C_{2}-R+\left(C_{2}+R\right) x\right)\right]
\end{gathered}
$$

There are two situations to be considered for developers according to Equation (5). One is that if $y=(S-A) / S$ $(S \geq A)$, thus $F\left(x^{*}\right)$ is 0 , implying all $x$ are trade-off solutions, and another is that if $y \neq(S-A) / S$ then $x^{*}{ }_{1}=0$ and $x_{2}^{*}=1$ while if $y>(S-A) / S$ the solution is $x_{1}^{*}=0$ and if $y<(S-A) / S$ then $x_{2}^{*}=1$. Accordingly, there are two situations to be considered for EPDs. One is that if $x=\left(R+C_{2}-C_{1}\right) /\left(C_{2}+R\right)$ and $\left(R+C_{2}=C_{1}\right)$, then $G(y)=0$ implying all $y$ are trade-off solutions, and another is that if $x \neq\left(R+C_{2}-C_{1}\right) /\left(C_{2}+R\right)$, then $y^{*}{ }_{1}=0$ and $y_{2}^{*}=1$, and when $R+C_{2} \geq C_{1} \quad\left(x<\left(R+C_{2}-C_{1}\right) /\right.$ $\left.\left(C_{2}+R\right)\right)$, then $y_{1}^{*}=0$ otherwise If $y_{2}^{*}=1$, and when $R+C_{2}<C_{1}\left(C_{1}-C_{2}-R+\left(C_{2}+R\right) x>0\right)$ then $G^{\prime}\left(y_{1}^{*}\right)>0, G^{\prime}\left(y_{2}^{*}\right)<0$ and $y_{2}^{*}=1$. If denoting $x_{0}=$ $\left(R+C_{2}-C_{1}\right) /\left(C_{2}+R\right), \quad y_{0}=(S-A) / S, \quad$ and $x_{0}>0$, $y_{0}>0$, we can understand the changing evolutionary ratios of developers and of EPDs (Figures 2-5).

Just as depicted in Figure 2 the strategies of EPDs and developers mainly depends on each other's choices of strategies, and further analyses made about Figure 3 and Figure 5 illustrates whether the fine is high or low the

Table 2. Payoff matrix by EPD and PGR-developers.

\begin{tabular}{cccc}
\hline & & \multicolumn{2}{c}{ EPD } \\
\hline \multirow{4}{*}{ CPGR-Developer } & No-monitoring & Monitoring \\
& Manag. & $\mathbf{0 , 0}$ & $\mathbf{0},-\boldsymbol{C}_{1}$ \\
& Non-manag. & $\boldsymbol{A},-\boldsymbol{C}_{2}$ & $\boldsymbol{A}-\boldsymbol{S}, \boldsymbol{R}-\boldsymbol{C}_{\mathbf{1}}$ \\
\hline
\end{tabular}

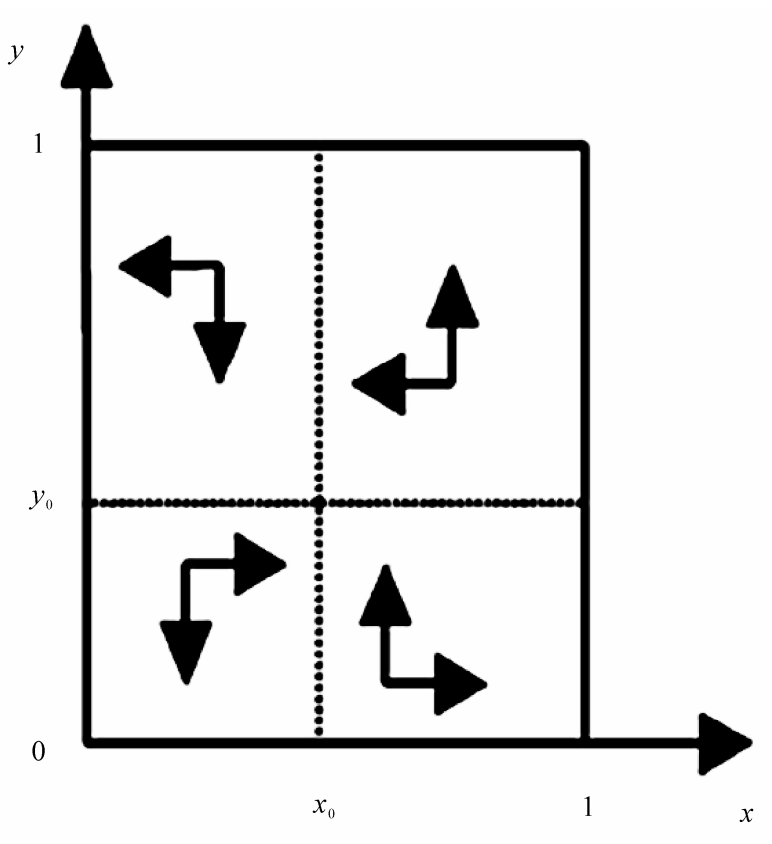

Figure 2. $S>A, R+C_{2}>C_{1}$.

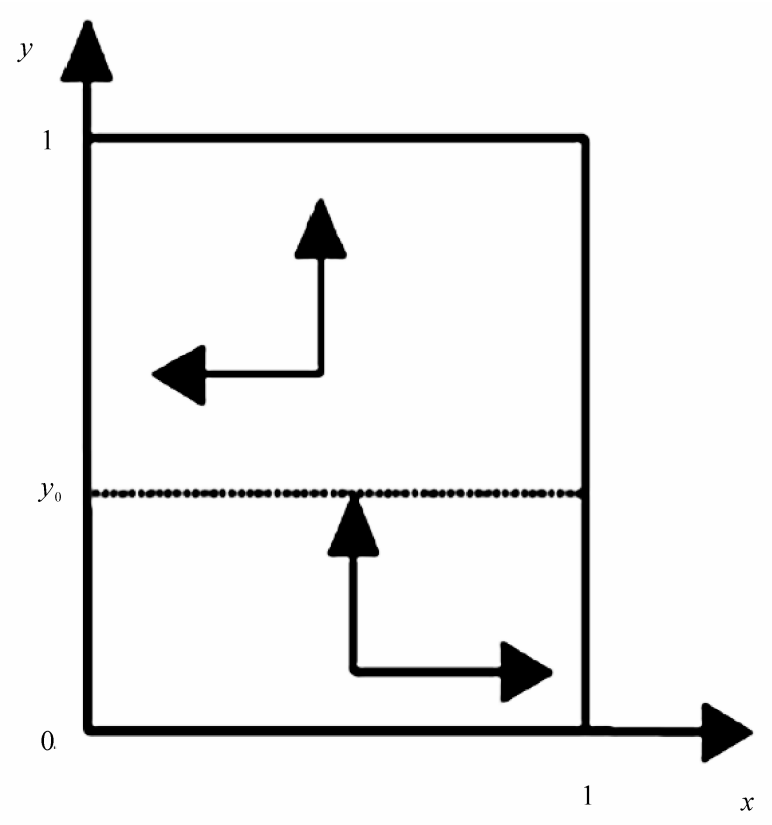

Figure 3. $S>A, R+C_{2}<C_{1}$.

trade-off of ESS between EPDs and developers is $x^{*}=0$, $y^{*}=1$. It means that the situation will arise when EPDs find higher monitoring costs with low incentives or rewards from government, or alternatively, it will occur when developers receive only small punishment with more green risk resources discovered by EPDs. Once achievement of game evolutionary, EPDs have to accept the non-monitor strategy and the developers accordingly take 


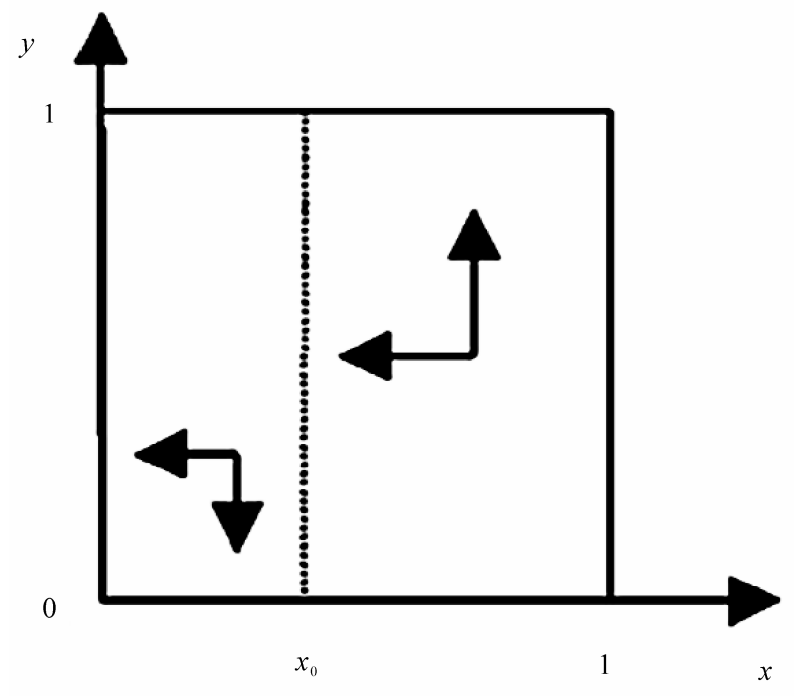

Figure 4. $S<A, R+C_{2}>C_{1}$.

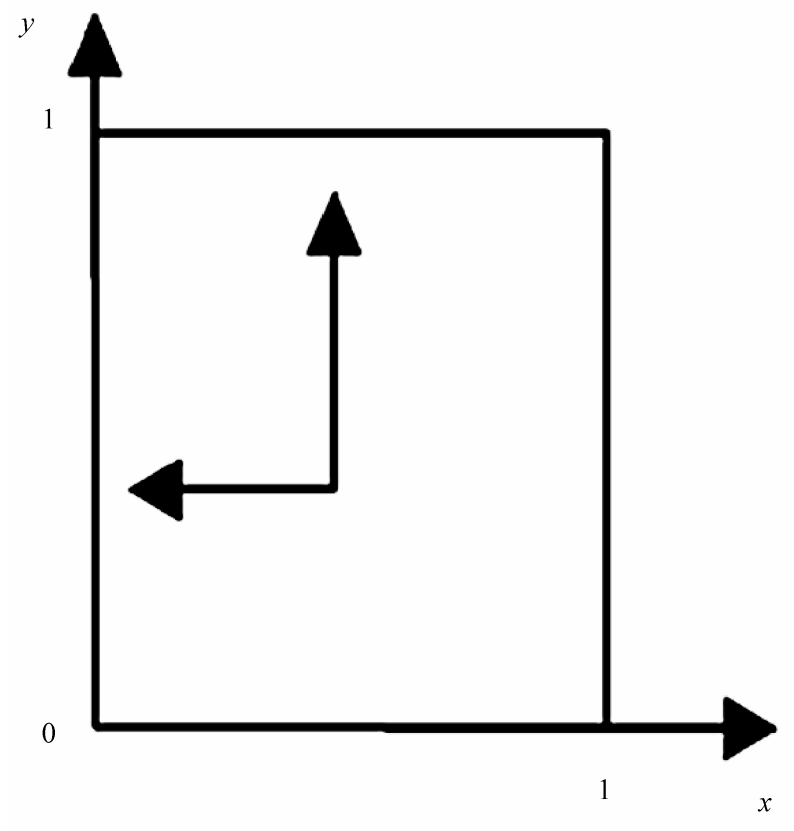

Figure 5. $S<A, R+C_{2}<C_{1}$.

actions of non-management strategy. However, from Figure 4 if $x^{*}=0, y^{*}=0$ which indicates that CPGRdevelopers will ignore green risks regardless of the monitoring of EPD because the fines from authorities are far from non-management's interest.

\section{Conclusions and Suggestions}

We can draw some conclusions from above analyses as follows:

1) Laws should be enacted to impose severe penalties on any non-management firm and its executives, thereby increasing the cost if a non-management strategy is followed;

2) In order to enhance environmental activism, government should exact strong punishment on those EPDs failing in their monitoring duties, and promote active environment protection enforcement;

3) The government should cover the costs of EPDs and raise incentives and rewards by investing in them and supplying new equipment. Such policies will accelerate uncovering non-complying developers;

4) New government policies mandating changes in the management mechanism of EPDs to function more easily, efficiently and profitably, will reduce reporting and accountability costs. Such polices would encourage EPDs to monitor CPGRs more closely and successfully.

\section{REFERENCES}

[1] J. X. Kasperson and R. E. Kasperson, "Global Environmental Risk," United Nations University Press, 2001.

[2] X. F. Zhang, "A New Challenge in Construction Management - the Sustainable Development," Resource, Environment and Projection, in Chinese, Vol. 9, 2004, pp. 88-91.

[3] B. V. Reddy and K. S. Jagadish, "Embodied Energy of Common and Alternative Building Materials and Technologies," Energy and Buildings, Vol. 35, No. 2, 2003, pp. 129-137.

[4] M. Lenzen and G. Treloar, "Embodied Energy in Buildings: Wood versus Concrete-Reply to Börjesson and Gustavsson," Energy Policy, Vol. 30, No. 3, 2002, pp. 249-255.

[5] C. Scheuer and A. Gregory, "Life Cycle Energy and Environmental Performance of a New University Building: Modelling Challenges and Design Implications," Energy and Buildings, Vol. 35, No. 10, 2003, pp. 1049-1064.

[6] A. Horvath, "Estimation of Environmental Implications of Construction Materials and Designs Using Life Cycle Assessment Techniques," Carnegie Mellon University, 1997.

[7] R. Wilson and A. Young, "The Embodied Energy PayBack Period of Photovoltaic Installations Applied to Buildings in the U.K.," Building and Environment, Vol. 31, No. 4, 1996, pp. 299-305.

[8] S. T. Li and H. Q. Li, "The Sustainable Development and the Dematerialization Construction in Construction Projects," Construction Economy, in Chinese, Vol. 4, 2007.

[9] H. Li, S. Q. He and S. T. Li, "Study on Synthesis Evaluation Model of Dematerialization Construction in Construction Projects," Construction Conserves Energy, in Chinese, Vol. 5, 2008, pp. 68-71.

[10] J. E. Young, et al., "Creating a Sustainable Materials 
Economy," State of the World, in Chinese, Vol. 6, 1995, pp. 88-92.

[11] Federal Minister for the Environment, "Towards Sustainable Development in Germany," Government of the Federal Republic of Germany, 1997.

[12] Y. H. Feng and Y. Q. Li, "Game Analyses of Environment Protection," Journal of Xi'an University of Science \& Technology, in Chinese, Vol. 23, No. 4, 2003, pp. 408- 410.

[13] W. Wu, G. Y. Cheng, H. C. Wang, et al., "The Gamble Analysis on Environmental Pollution Problem," Sys- tems Engineering-Theory \& Practice, in Chinese, Vol. 21, No. 10, 2001, pp. 115-119.

[14] D. M. Wang and W. Q. Li, "Application of Game Theory to Environmental Protection," Urban Environment \& Urban Ecology, in Chinese, Vol. 17, No. 5, 2004, pp. 45-47.

[15] H. Gintis, "Game Theory Evolving," Princeton University Press, Princeton, 2000.

[16] S. Y. Xie, "Economic Game Theory," in Chinese, Fudan University Press, Shanghai, 2002. 